Research Article

\title{
Neutron Transport Simulations of RBMK Fuel Assembly Using Multigroup and Continuous Energy Data Libraries within the SCALE Code
}

\author{
Andrius Slavickas (iD, Raimondas Pabarčius, Aurimas Tonkūnas, and Sigitas Rimkevičius \\ Lithuanian Energy Institute, Breslaujos g. 3, LT-44403 Kaunas, Lithuania \\ Correspondence should be addressed to Andrius Slavickas; andrius.slavickas@lei.lt
}

Received 11 December 2020; Accepted 28 February 2021; Published 10 March 2021

Academic Editor: Peter Ivanov

Copyright (c) 2021 Andrius Slavickas et al. This is an open access article distributed under the Creative Commons Attribution License, which permits unrestricted use, distribution, and reproduction in any medium, provided the original work is properly cited.

\begin{abstract}
The neutron transport simulations of RBMK-1500 fuel assembly were performed using both multigroup and continuous energy data libraries available within the SCALE code system in order to validate its suitability for the estimation of RBMK neutronic characteristics. The resonance processing of cross section, involved in the preparation of the multigroup data library, has a significant impact on neutron transport calculations. Standard Dancoff factors (DFs) used for the heterogeneous geometry of RBMK fuel assembly are insufficient for the accurate estimation of resonance self-shielding. Thus, the SCALE module MCDancoff was used in this study to determine location-specific DFs. The results of RBMK-1500 fuel assembly simulations using standard and user-defined DFs were compared. In addition, the continuous energy (CE) cross-section data library was applied for the benchmark calculations. The impact of different nuclear data libraries on neutron transport simulations was tested as well. It was found out that the usage of the multigroup data libraries generates some deviation from the reference simulations obtained with CE libraries. The CE library based on the estimated ENDF/B-VII.1 data proved to be the best alternative for neutron transport simulations of RBMK fuel assembly.
\end{abstract}

\section{Introduction}

The objective of this paper is to validate the suitability of the SCALE code for estimation of RBMK reactor nuclear fuel neutronic characteristics and to propose the best suitable models and factors to be used in the calculations. The SCALE code [1] provides a suite of computational tools of criticality safety analysis that is primarily based on the KENO Monte Carlo module for the neutron transport simulations to calculate the neutron multiplication factor on both multigroup (MG) and continuous energy (CE) modes. The deterministic T-NEWT module, available within the SCALE, is applicable with an MG data library only; thus, the KENO Monte Carlo module was chosen for the analysis.

All cross-section libraries available within the SCALE code were processed from ENDF/B evaluated data libraries. The CE and several MG cross-section data libraries with several group structures are available in the SCALE for neutron transport calculations, so that a user may select the nuclear data library based on a specific application, required accuracy, or preferable execution time. The pointwise $\mathrm{CE}$ v7.0 (based on ENDF/B-VII.0 data) and CE v7.1 (based on ENDF/B-VII.1 data) libraries were employed. The CE data are further processed into the MG data. The 238-group v7238 (ENDF/B-VII.0) and the 252-group v7-252 (ENDF/BVII.1) libraries were employed in this study's simulations as well.

A generation of problem-specific MG cross sections is needed for performing the neutronic analysis if MG mode is chosen. These cross sections are significantly affected by the resonance self-shielding, the effect of which can be classified into two types regarding the cause: energy self-shielding and spatial self-shielding. The spatial self-shielding is primarily induced by heterogeneous effects of the reactor core or fuel 
assembly. Different materials and their locations can influence significant changes in neutron fluxes over short distances. Thus, the right estimation of spatial self-shielding is important for every specific problem related to heterogeneous geometry.

In the reactors' cores operated on thermal-neutrons, the fuel assemblies are composed of fuel rods surrounded by the moderator and control rods. Thus, the primary basic element of the core is a fuel pin cell. The neutrons, which are born in the fuel pin, are slowed down in the moderator, while some of them are absorbed in the fuel, and some neutrons can enter another fuel pin. The Dancoff factor (DF) [2] is used in neutron transport calculations in order to estimate resonance self-shielding effects between neighboring fuel pins. DF indicates the probability that a neutron from the surface of one fuel pin will pass through the external media and will enter a nearby located fuel pin.

On the assumption that the SCALE lattice cell treatment assumes that a fuel pin lies in an infinite lattice, which composes identical fuel pins with the same pitch, an analytical expression for DF is employed and applied uniformly throughout the lattice [1]. However, reactor cores usually are composed of finite fuel assemblies with some distance between them. Additional moderation usually exists in these gaps between fuel assemblies, and it increases self-shielding effects between fuel pins. Thus, the application of standard DF underestimates the actual self-shielding effects. Even more, because of resonance self-shielding, the effect of this additional moderation decreases from outer to inner fuel pins. More precisely, location-specific DFs can be calculated using the SCALE module MCDancoff and introduced to neutron transport calculations [3]. This possibility was examined quantitatively in this paper.

The best-known example of heterogeneous fuel assemblies is BWR, where nonboiling water between fuel assemblies and in special water channels within assemblies plays the part of the additional moderator to otherwise lowmoderated assembly. Previous studies demonstrated that the application of location-specific DFs estimated using the MCDancoff module has a significant impact on BWR neutron transport calculations [3-5]. For example, it was assessed that the reactivity difference for GE14 assembly irradiation simulations using both standard and locationspecific DFs was in the range of $800-1100 \mathrm{pcm}$ [3]. Therefore, the location-specific DF is usually applied in the case of BWR fuel assemblies' simulations [6-9].

Due to high geometry heterogeneity, RBMK assemblies can be treated similar to BWR. Fuel pins inside the assembly are cooled by water, which can be considered as a moderator as well. However, the primary moderator in RBMK reactors is graphite, which is positioned outside the pressure tube. Only a coolant in a fuel pitch cell is considered as a moderator in the estimation of standard DFs for RBMK assemblies, while the influence of graphite as a primary moderator is not considered. Thus the resonance processing with standard DFs is not sufficient for the accurate estimation of MG cross sections.

The coupling between neutron transport and fuel burn-up simulations is undeniable and strong, as accurate neutronics data are required to simulate the radionuclide inventory. The results of neutron transport simulations are substantial for correct prediction of the uranium depletion, the plutonium production, and the build-up of fission products. The previous study [10] attempted to estimate the influence of additional graphite moderation on the isotopic fuel composition by increasing the lattice pitch (the amount of coolant/moderator as well) from $1.605 \mathrm{~cm}$ to $2.5 \mathrm{~cm}$. It was found that the differences in the actinide inventory could reach $10-15 \%$.

This paper presents the results of RBMK-1500 fuel assembly neutrons transport simulations employing two sets of DFs: standard and user-defined. No resonance processing is needed and the cross sections are used directly if the CE calculation mode is selected. Thus, the simulation with the applied CE cross-section data library was performed for the benchmarking purpose of both resonance processing models. In addition, the impact of the updated cross section from ENDF/B-VII.1 evaluated data on the neutron transport simulation results was analyzed. The discrepancies of reactivity between simulations are demonstrated and explained.

\section{Computational Methodology}

Based on the knowledge and experience gained by the authors on RBMK, the fuel assembly of the RBMK-1500 reactor was chosen as the object of investigation. Till June 1995, the Ignalina NPP operated on fuel assemblies with $2 \%$ $\mathrm{U}^{235}$ enrichment uranium only. Since the majority of spent nuclear fuel at Ignalina NPP consists of these types of fuel assemblies, the simulations were executed for this fuel type. The impurities of the initial fuel composition for $\mathrm{U}^{234}$ and $\mathrm{U}_{236}$ are $0.021 \%$ and $0.0018 \%$, respectively.

The RBMK-1500 fuel assembly consists of 18 fuel rods arranged in two concentric rings (Figure 1) with the central carrier rod [11]. In the inner ring, there are 6 equally spaced fuel rods while in the outer ring, there are 12 equally space fuel rods. Fuel rods are packed with cylindrical uraniumdioxide pellets, outside diameter, $1.152 \mathrm{~cm}$. The fuel pellets are placed into the clad tube of zirconium alloy, the outside diameter and the wall thickness of which is $1.36 \mathrm{~cm}$ and $0.9 \mathrm{~mm}$, respectively. These tubes are pressurized with helium and sealed. The fuel assemblies are located within a rectangular-form graphite block $25 \times 25 \mathrm{~cm}$.

The zirconium alloy content used for cladding and the pressure tube is different. $\mathrm{Zr}$ and $\mathrm{Nb}$ as cladding materials account for $98.9 \%$ and $1 \%$, respectively. Meanwhile, in the pressure tube, they account for $97.4 \%$ and $2.5 \%$ of the alloy. Other impurities that included zirconium alloys are $\mathrm{Hf}^{178}$ (0.04\%), Fe (0.018\%), $\mathrm{Ni}(0.018 \%)$, and $\mathrm{Al}(0.018 \%)$. SS3304 stainless steel is used as a grid element.

In order to maintain the same amount of fissile nuclear material as is prescribed in the design, the theoretical fuel density was reduced. The fuel temperature was set at $1000 \mathrm{~K}$, while the temperatures of coolant, structural, and graphite materials were set at $557 \mathrm{~K}, 575 \mathrm{~K}$, and $750 \mathrm{~K}$, respectively, in the simulations to represent the average operation conditions. The RBMK-1500 reactor core has a heterogeneous axial void distribution across fuel assembly, and the average coolant density of $0.43 \mathrm{~g} / \mathrm{cm}^{3}$ was considered for the analysis. 


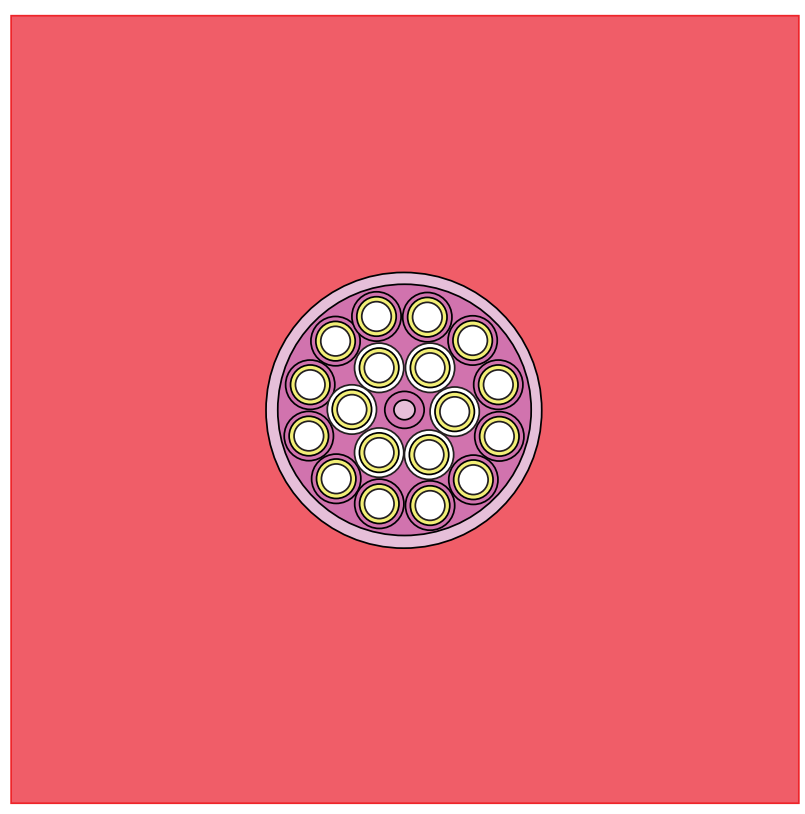

FIgUre 1: The model of RBMK-1500 fuel assembly.

SCALE 6.2.3 code package [1] was used to perform the neutron transport calculations. The verification and validation of the code package are based on the experimental investigations of RBMK-1000 fuel [12, 13]. The SCALE package provides a framework with 89 computational modules that could be selected on the desired solution strategy. The KENO-VI sequence was employed for Monte Carlo neutrons transport simulations in this study. 36 million neutron histories were used for the reliable estimation of the neutron multiplication factor. The cross section of the $3 \mathrm{D}$ model representing the RBMK assembly placed in the pressure tube channel and surrounded by the graphite block was used in simulations (Figure 1).

The first set of simulations was performed with the application of standard DF. DFs were calculated automatically using the SCALE lattice treatment within the TRITON sequence. The automatic lattice treatment assumes an infinite lattice that consists of identical fuel pin cells segregated inside the regions for fuel, gap, cladding, and moderator. The region of graphite block outside fuel assembly and fuel pin cells was not included during the estimation of spatial resonance shelf-shielding effects. The triangular pitch with the size of $1.605 \mathrm{~cm}$ was used for the resonance self-shielding calculations and the preparation of problem-specific multigroup cross sections. It was estimated that the standard DFs, which are identical for all fuel pins in the assembly, are equal to 0.5685 .

The second set of simulations was performed with location-specific DFs, which were manually inserted into the input file of TRITON. The MCDancoff module within the SCALE code was used to calculate DFs for specific locations in the fuel assembly. The calculation within the MCDancoff module involves the following paths of neutrons throughout all regions of the system and materials until they are absorbed or exit the system [1]. Thus, all details of geometry and materials of the heterogeneous system were considered for the estimation of spatial resonance self-shielding effects. Identical geometry setup as in KENO-VI sequence was used in MCDancoff simulation. MCDancoff module uses a onegroup xn01 library to calculate DFs. 100 generations with 300 neutrons per generation were used to produce DFs.

As shown in Figure 2, determined DFs could be grouped into two groups since similar values were calculated for fuel pins in the inner and outer rings. The differences in DF values between fuel pins of the same ring are related to the statistical error, which comes with the Monte Carlo method. Hence, two averaged DFs values were determined and used for the following neutron transport simulations: 0.452 for the inner ring and 0.289 for the outer ring. DFs for fuel pins in the inner ring are larger since the effect of additional moderation from the graphite block for the inner ring fuel pins is shielded by fuel pins of the outer ring. The user-defined DFs are smaller than standard DFs for inner and outer rings by $21 \%$ and $49 \%$, respectively. It means that the actual effect of spatial resonance self-shielding is larger than the one estimated using the automatic lattice cell treatment. The SCALE lattice treatment increased the triangular pitch of infinite lattice from $1.605 \mathrm{~cm}$ to $1.775 \mathrm{~cm}$ (inner ring) and $2.11 \mathrm{~cm}$ (outer ring) to get the same values as of user-defined DFs.

The third simulation set was performed using the CE data libraries. Since the resonance processing is not needed if a CE cross-section library is specified, the simulations with a specified CE mode can be used as a benchmark for the evaluation of the resonance processing models in cases where the MG mode was specified.

\section{Discussion of Results}

The neutron multiplication factor is one of the essential neutronic characteristics derived from the neutron transport simulations. The discrepancies in values of multiplication factor estimated using the SCALE code for the transport simulation of RBMK-1500 fuel assembly considering the different options for the cross-section processing and available cross-section data libraries are presented in this section. The reasons behind these discrepancies are disclosed as well.

3.1. The Influence of User-Defined DFs. As was mentioned before, the use of multigroup cross-section libraries requires the resonance processing of cross-section data. An infinite lattice, which consists of identical fuel pin cells, is considered for the estimation of standard DF (sDF) values within the SCALE code during an automatic lattice treatment without any user interference. However, the user has another possibility to define manually $\mathrm{DF}(\mathrm{uDF}$ ) values, which are estimated considering the real geometry of the fuel cell. For both cases, the simulations were performed and neutron multiplication factors were compared.

The neutron transport simulations were made using both v7-238 and v7-252 multigroup cross-section data libraries available in the SCALE code; the results are depicted in Figure 3. It is seen that the multiplication factor determined using $\mathrm{sDF}$ has $0.5 \%$ and $0.36 \%(455 \mathrm{pcm}$ and $317 \mathrm{pcm})$ higher values for v7-238 and v7-252 data libraries, 


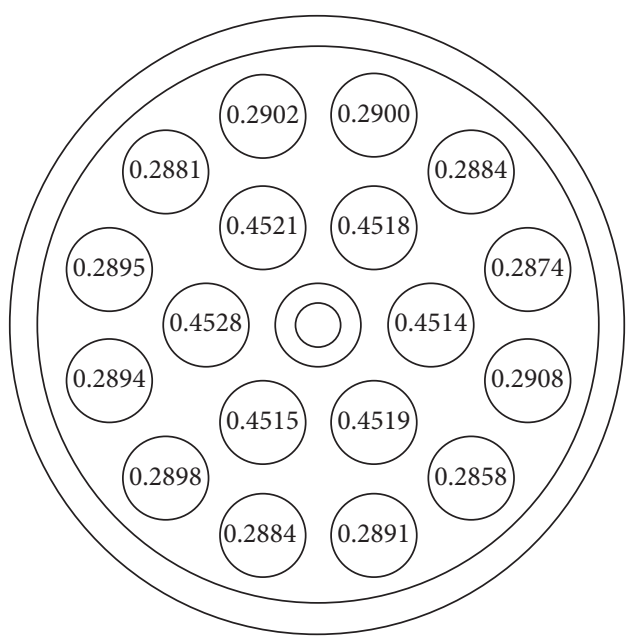

FIgURE 2: Location-specific DFs calculated by the MCDancoff module (RBMK-1500 case).

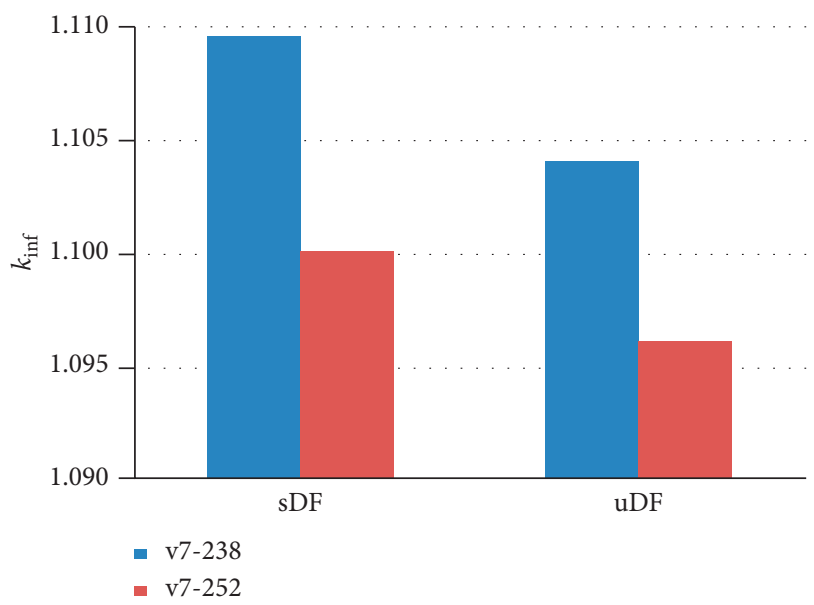

Figure 3: Neutron multiplication factor for different MG mode simulations (RBMK-1500, fresh $2 \% \mathrm{U}^{235}$ fuel).

respectively, in comparison with simulations using uDF. It is evident that the change of the multiplication factor is reflected by the redistribution of neutron absorption in separate regions.

Representative results of fractional absorption data for all simulation cases, including ones with CE libraries, are shown in Figure 4. It is seen that the majority of neutrons are absorbed by fuel pins (in inner $23 \%$, in outer 53\%). Other neutrons (20\%) are absorbed in coolant and graphite block and additionally are lost due to the parasitic absorption in other structure materials (4\%). Slight differences between fractional absorption data in some geometry regions (Figure 4) should explain the discrepancies in the calculated multiplication factors.

The decomposition analysis of the neutron multiplication factor, being an extremely useful instrument and quantitative testing measure [14], was employed in this study with the objective to explain the discrepancies in simulation results. The reactivity differences between simulation cases were decomposed to separate components, which represent the contributions of the separate region to the total reactivity difference. The total reactivity change $\Delta \rho$ through separate components $i$ can be expressed as follows:

$$
\Delta \rho=\sum_{i} \Delta \rho_{i}=\Delta \sum_{i}\left(-\frac{a_{i}}{k_{\text {inf }}}\right)=\sum_{i} \frac{a_{i, x} * k_{\text {inf., } y}-a_{i, y} * k_{\text {inf., } x}}{k_{\text {inf. } x} * k_{\text {inf., } y}} .
$$

Here, indexes $x$ and $y$ stand for different simulation cases. Meanwhile, $k_{\text {inf }}$ and $a_{i}$ are the infinite neutron multiplication factor and the fractional absorption for component $i$, respectively. For more details, see [15].

The results of the decomposition analysis examining the reactivity difference $\left(\Delta \rho_{\mathrm{SDF}-\mathrm{uDF}}\right)$ between $\mathrm{sDF}$ and $\mathrm{uDF}$ cases and employing v7-238 and v7-252 MG libraries are shown in Figure 5 . It is seen that $\Delta \rho_{\mathrm{sDF}-\mathrm{uDF}}$ are mainly influenced by fuel regions, predominating clearly by fuel pins located in the outer ring. The outer fuel pins are affected more significantly in case of the usage uDF since the additional moderation (graphite block) influences greater neutronic changes in comparison to the effect in inner fuel pins. The positive $\Delta \rho_{\mathrm{sDF}-\mathrm{uDF}}$ in fuel regions is related to the slight increase in fractional absorption. Neutron absorptions increase from $76.74 \%$ for the sDF case to $76.84 \%$ for the $\mathrm{uDF}$ case if v7-238 data library was applied. Meanwhile, it increases from $76.19 \%$ to $76.26 \%$ with the use of v7-252 data library (see Figure 4).

The fractional absorption spectra (Figure 6(a)) were used to estimate $\Delta \rho_{\mathrm{sDF}-\mathrm{uDF}}$ in each energy group (Figure $6(\mathrm{~b})$ ) on purpose to explain the total $\Delta \rho_{\mathrm{sDF}-\mathrm{uDF}}$ in outer fuel pins. It is seen that although most neutrons are absorbed in the ranges of thermal and epithermal energy $(0.01-0.5 \mathrm{eV})$, the differences in reactivity are mainly influenced by the change in fractional absorption in the ranges of resonance and fast energy $(6 \mathrm{eV}-13 \mathrm{MeV})$.

The only discrepancy found between $\mathrm{sDF}$ and $\mathrm{uDF}$ cases was different DFs values used for the resonance processing of cross section. Lesser $\mathrm{uDF}$ than $\mathrm{sDF}$ values define a lower resonance escape probability as more neutrons are absorbed in fuel pins before they are slowing down to thermal energies (reflected in Figures 5 and 6(b)). Thus, the consideration of fuel pin location in the RBMK-1500 assembly and both geometry and material details for uDF estimation, as well as the follow-up application of a more detailed resonance processing model with $\mathrm{uDF}$ values, give reasons for higher resonance absorption in fuel and a lower neutron multiplication factor.

3.2. Transport Simulations: $C E$ versus $M G$. If the CE crosssection data library is specified for neutron transport simulations, then the resonance processing of cross-section data is not needed, and such CE simulation can be used to evaluate the accuracy of the resonance processing models used in MG cross-section library applications. The comparison of neutron multiplication factor for $\mathrm{CE}$ and $\mathrm{uDF}$ cases using both ENDF/B-VII. 0 and ENDF/B-VII. 1 data was performed and the results are depicted in Figure 7. An almost ideal match was obtained between $\mathrm{CE}$ and uDF cases, 


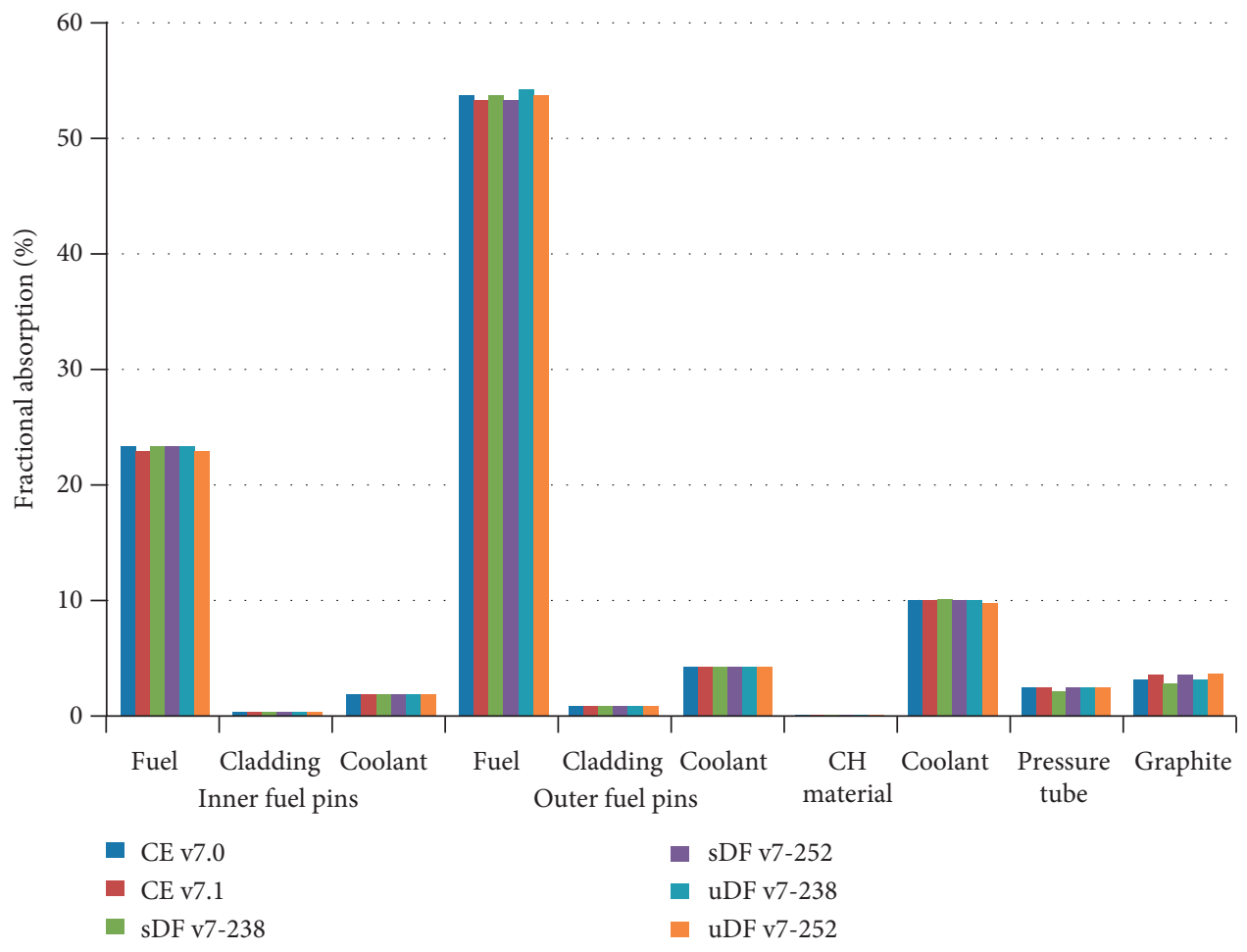

FIgURE 4: Fractional absorption values for separate regions.

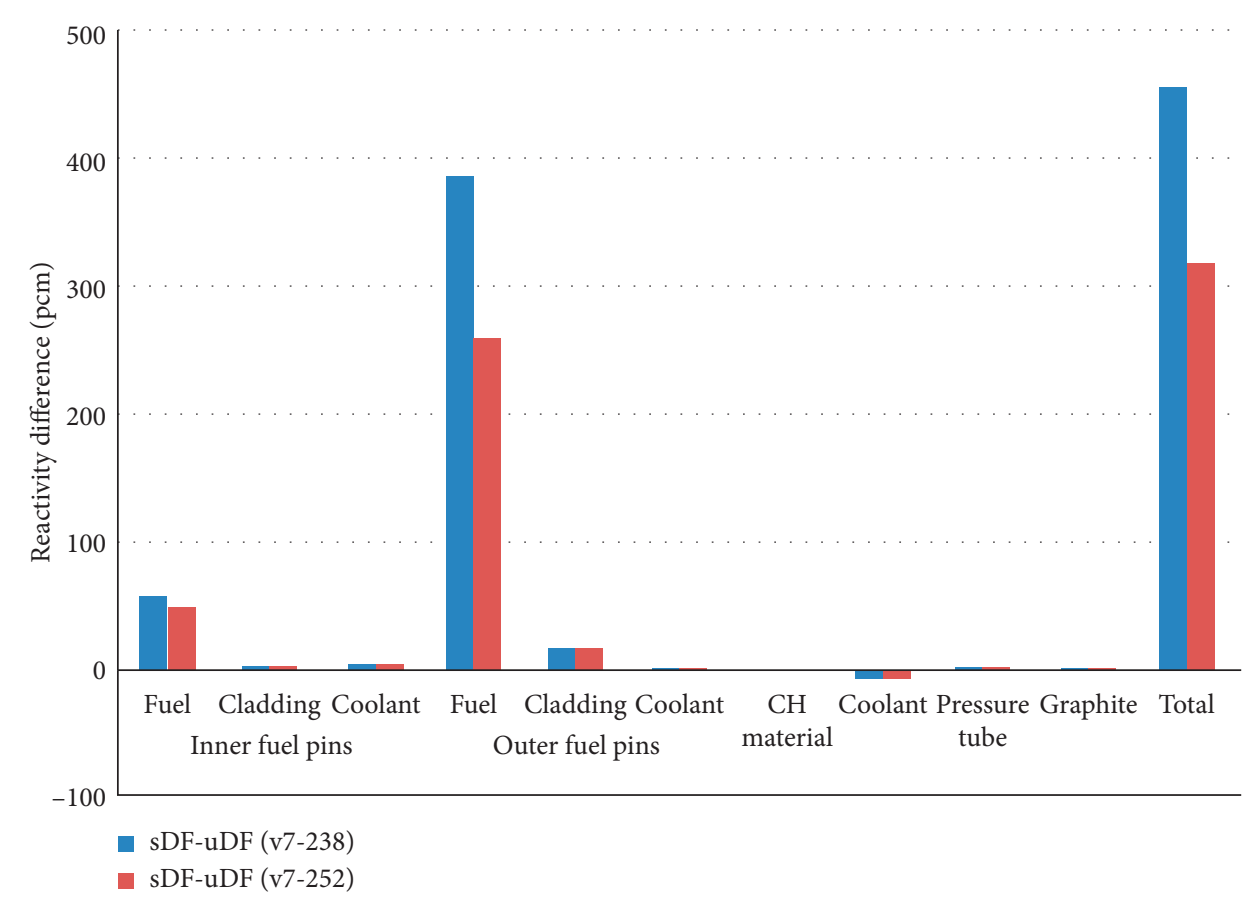

FIGURE 5: $\Delta \rho_{\text {SDF-uDF }}$ for separate geometry regions.

the difference being only $0.07 \%(60 \mathrm{pcm})$, in the case of the ENDF/B-VII. 0 library. Meanwhile, when applying ENDF/ B-VII. 1 data, the multiplication factors differ by $0.25 \%$ $(226 \mathrm{pcm})$.

The results of decomposition analysis, analyzing the differences in reactivity between $\mathrm{CE}$ and $\mathrm{uDF}$ cases $\left(\Delta \rho_{\mathrm{CE}-}\right.$ uDF) in separate regions (Figure 8 ), identify some discrepancies in fractional absorption in fuel and pressure tube regions. The changes of fractional absorptions in fuel regions (mainly in inner fuel pins) influence positive $\Delta \rho_{\text {CE- }}$ uDF values, while the changes in the pressure tube region influence negative $\Delta \rho_{\text {CE-uDF }}$ values. Nevertheless, the relative shift of fractional absorption in fuel regions is larger than that in the pressure tube region; thus, the total $\Delta \rho_{\mathrm{CE}-\mathrm{uDF}}$ is 


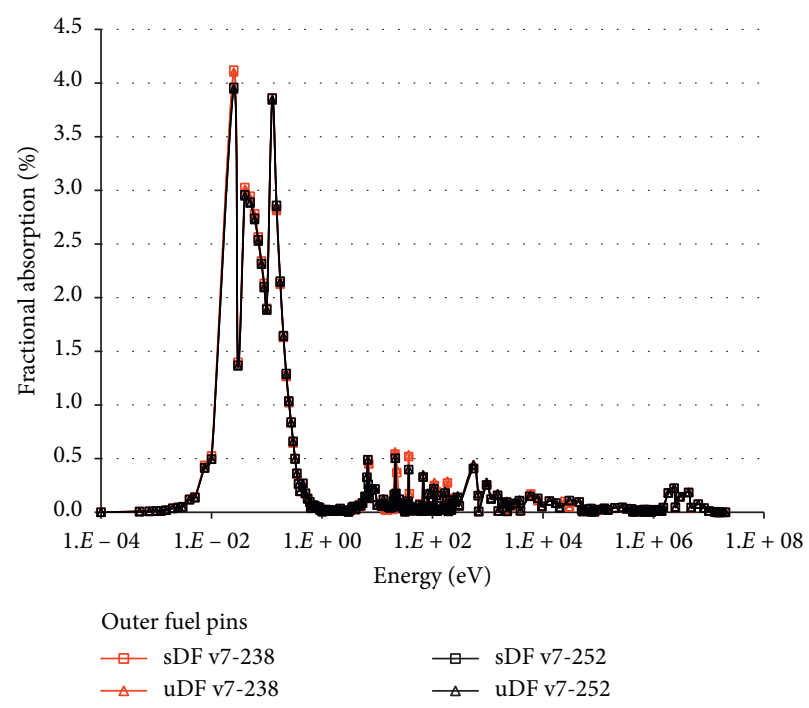

(a)

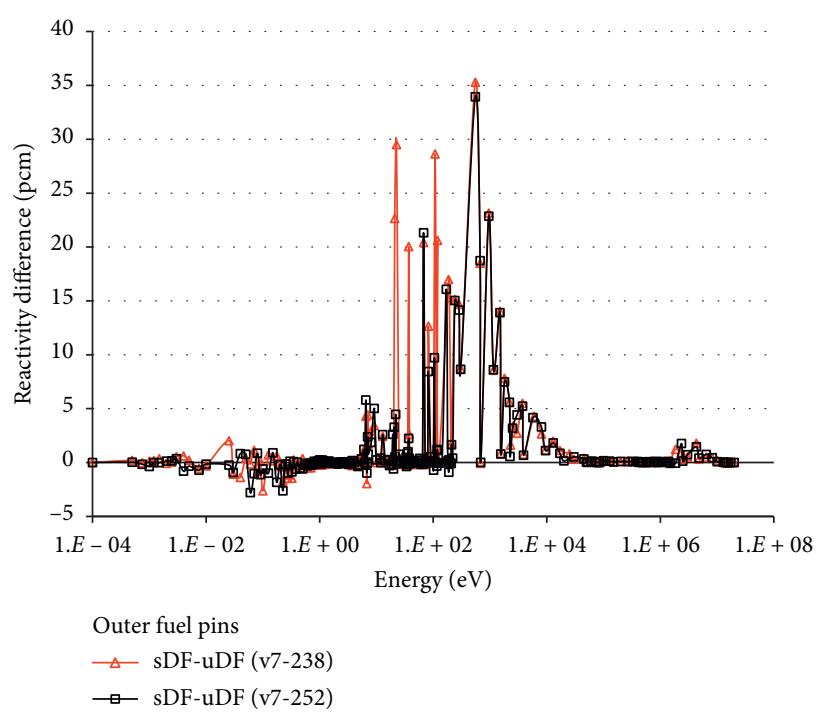

(b)

Figure 6: Distributions of fractional absorption (a) and $\Delta \rho_{\text {sDF-uDF }}$ (b) in whole energy range (outer fuel pins).

positive. Though the total difference is relatively low $(60 \mathrm{pcm})$ in the case of applied ENDF/B-VII. 0 data library, it increases 4 times (to $236 \mathrm{pcm}$ ) when simulations were run with ENDF/B-VII. 1 data library.

The distribution of $\Delta \rho_{\mathrm{CE}-\mathrm{uDF}}$ in the whole energy range in main contributing regions, that is, outer fuel pins and pressure tube, is depicted in Figure 9. The simulation results obtained using the ENDF/B-VII. 1 data library are only presented as the fractional absorption data were available only for $\mathrm{CE}$ cases with a structure of 252 energy groups; therefore, MG libraries of the same structure were used for the simulation of uDF cases. Following performed decomposition analysis of $\Delta \rho_{\mathrm{CE}-\mathrm{uDF}}$ into separate energy groups revealed the various changes in fractional absorptions.

The main changes in $\Delta \rho_{\mathrm{CE}-\mathrm{UDF}}$ in the pressure tube region (Figure 9(a)) occur at resonance peaks. $\mathrm{Hf}^{178}$ resonance peak $\sim 8 \mathrm{eV}(-49 \mathrm{pcm}), \mathrm{Zr}^{90}$ resonance peaks $\sim 188 \mathrm{eV}$ $(-16 \mathrm{pcm})$, and $\sim 305 \mathrm{eV}(-24 \mathrm{pcm})$ are very clear and mainly contribute to the negative $\Delta \rho_{\mathrm{CE}-\mathrm{uDF}}$. The negative $\Delta \rho_{\mathrm{CE}-\mathrm{uDF}}$ means that the fractional absorption for the uDF case is higher compared to the CE case. Thus, the postprocessed resonance cross sections of the pressure tube in the MG cases are clearly overpredicted, and, for example, at resonance peaks of $\mathrm{Hf}^{178}$ and $\mathrm{Zr}^{90}$, the level of such overprediction exceeds $25-40 \%$ (Figure 10). Obviously, the same overprediction of resonance cross sections occurs for cladding material too, because the cladding composition is similar to the pressure tube composition ( $\mathrm{Zr}$ alloy with different $\mathrm{Nb}$ concentration). However, the estimated $\Delta \rho_{\text {CE-uDF }}$ for cladding regions are smaller (Figure 8 ) since the total mass, and the effect of the cladding on the neutron transport processes is relatively smaller compared to the pressure tube.

The results of the analysis showed that most changes of $\Delta \rho_{\mathrm{CE}-\mathrm{uDF}}$ in the fuel pin region occur at the same resonance peaks and in the same energy ranges as it is for the pressure

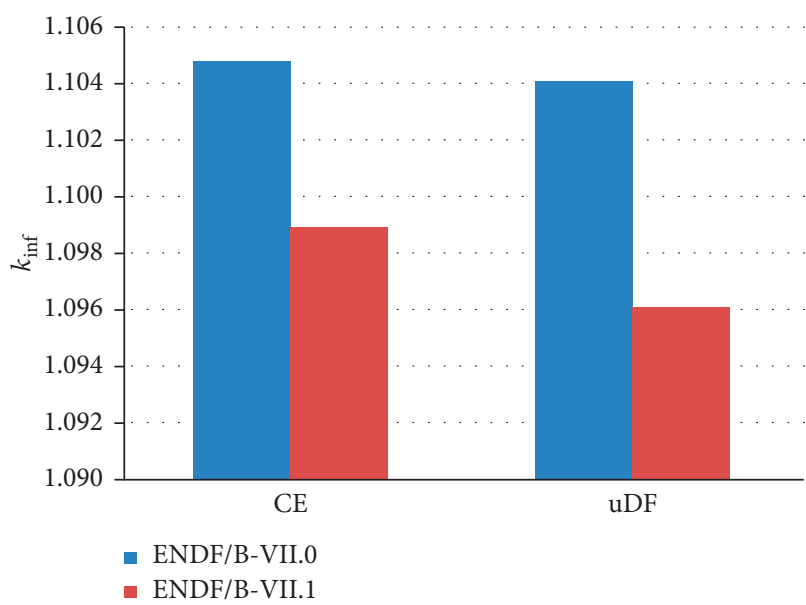

FIGURE 7: Neutron multiplication factor: comparison of CE and uDF cases.

tube region (Figure 9(b)). Thus, the different neutron absorption rate in the pressure tube and cladding regions influences the perturbations of neutron flux in the fuel pin regions. Although the changing of neutron flux in the thermal neutron range $(<1 \mathrm{eV})$ calls a significant increase of $\Delta \rho_{\text {CE-uDF }}$ in fuel regions $(-34 \mathrm{pcm}$ at the peak $\sim 0.2 \mathrm{eV}$ for outer fuel pins, Figure 9(b)) due to larger absorption cross sections, $\Delta \rho_{\mathrm{CE}-\mathrm{uDF}}$ in the resonance and fast energy range $(>1 \mathrm{eV})$ results in a positive $\Delta \rho_{\mathrm{CE}-\mathrm{uDF}}$ in fuel regions, which compensates for and exceeds the negative $\Delta \rho_{\mathrm{CE}-\mathrm{uDF}}$ obtained in the pressure tube region.

There are $\Delta \rho_{\mathrm{CE}-\mathrm{uDF}}$ in the neutron energy range of $20-120 \mathrm{eV}$, which cannot be explained by changes in neutron flux (Figure 9(b)). These differences should be only related to the inaccuracy during resonance processing of cross sections. As can be seen in Figure 5, the more detailed resonance processing model with $\mathrm{uDF}$ gives larger $\Delta \rho_{\mathrm{CE}-\mathrm{uDF}}$ 


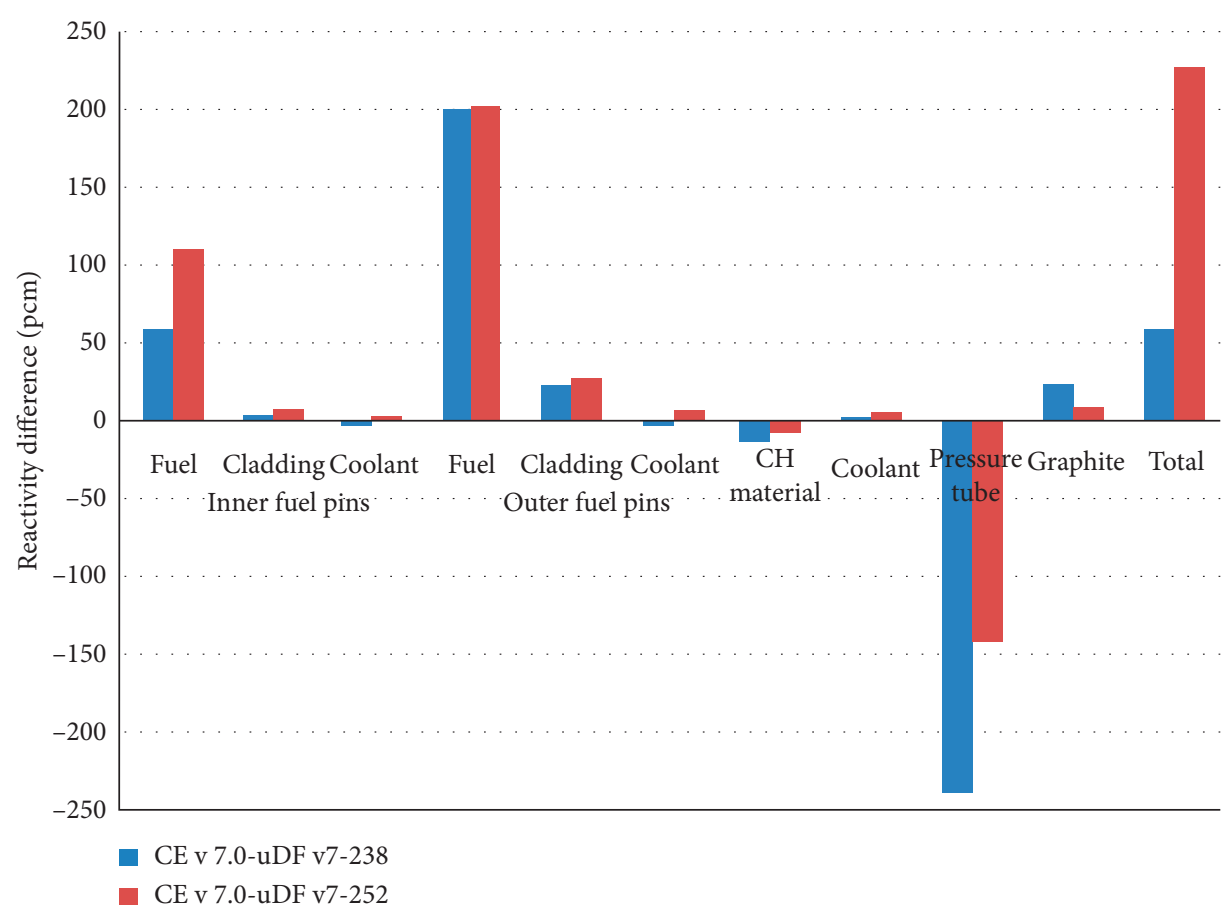

FIGURE 8: $\Delta \rho_{\text {CE-uDF }}$ cases for separate regions.

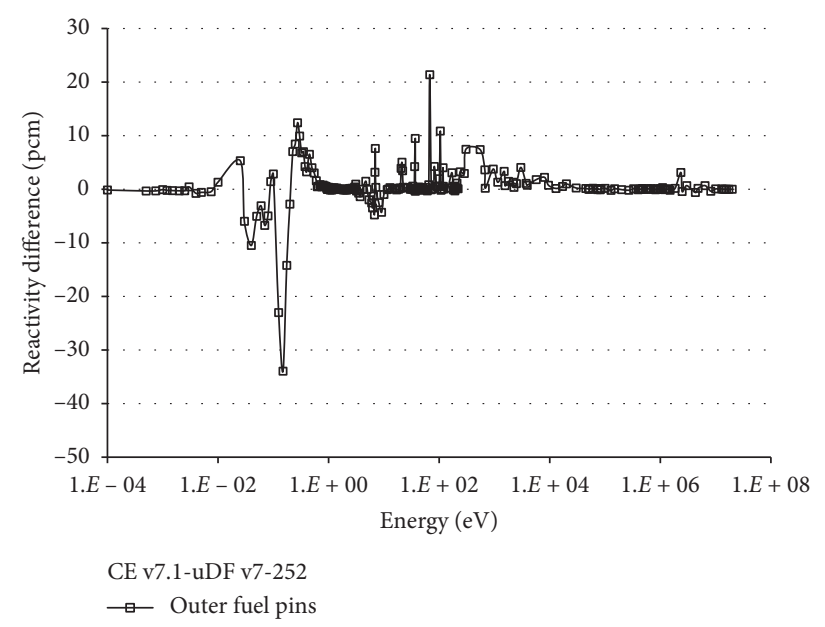

(a)

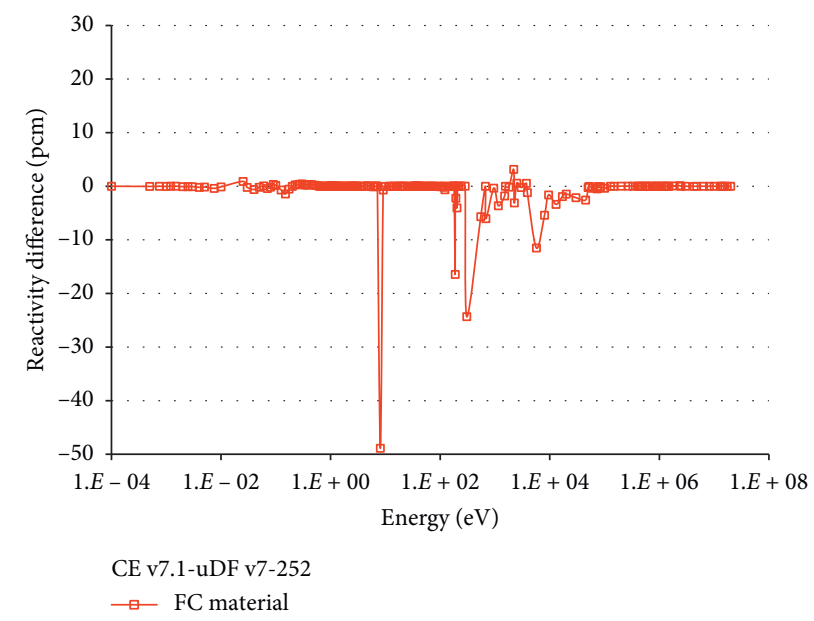

(b)

FIgURE 9: $\Delta \rho_{\mathrm{CE}-\mathrm{uDF}}$ for pressure tube (a) and outer fuel pins (b) regions.

in the case of 238-group data library application in comparison to the case with 252-group data library. The defined larger $\Delta \rho_{\mathrm{CE}-\mathrm{uDF}}$ can be explained by the remaining discrepancies in $\Delta \rho_{\text {CE-uDF }}$ in the mentioned $20-120 \mathrm{eV}$ energy range (see Figure 6(b)), while the 252-group data library gives significantly lower $\Delta \rho_{\mathrm{CE}-\mathrm{uDF}}$ at this energy range. Thus, it can be argued that the resonance processing of cross section is more accurate with the application of 238 group data library for transport simulations as such an approach allows the calculation of reactivity values closer to the $\mathrm{CE}$ case reference values.

3.3. The Impact of Updated Cross-Section Data Library. All cross-section libraries available within the SCALE code were processed from ENDF/B-VII. 0 and ENDF/B-VII. 1 evaluated data files. The comparison of neutron multiplication factor calculated using v7-238, CE v7.0, and v7-252, CE v7.1 libraries is depicted in Figure 11. It is evident that the 


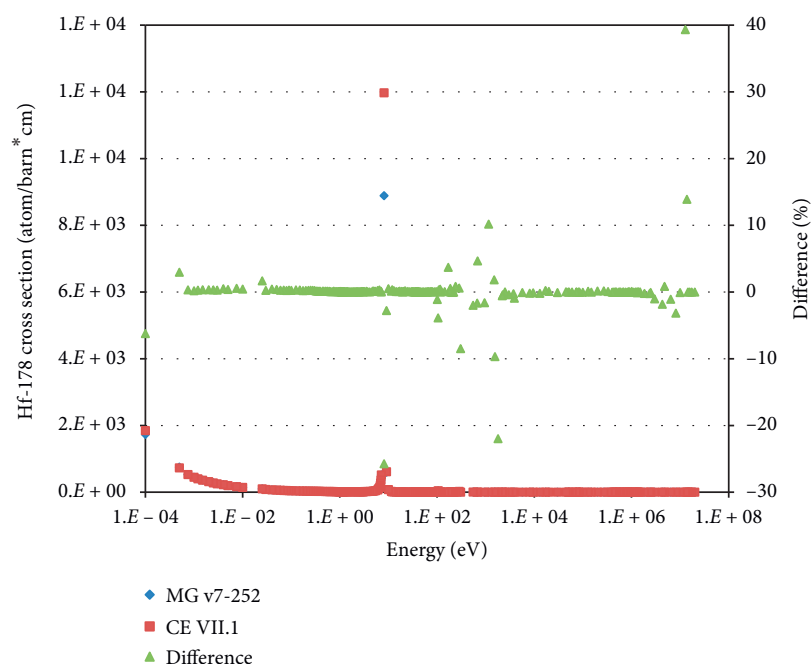

(a)

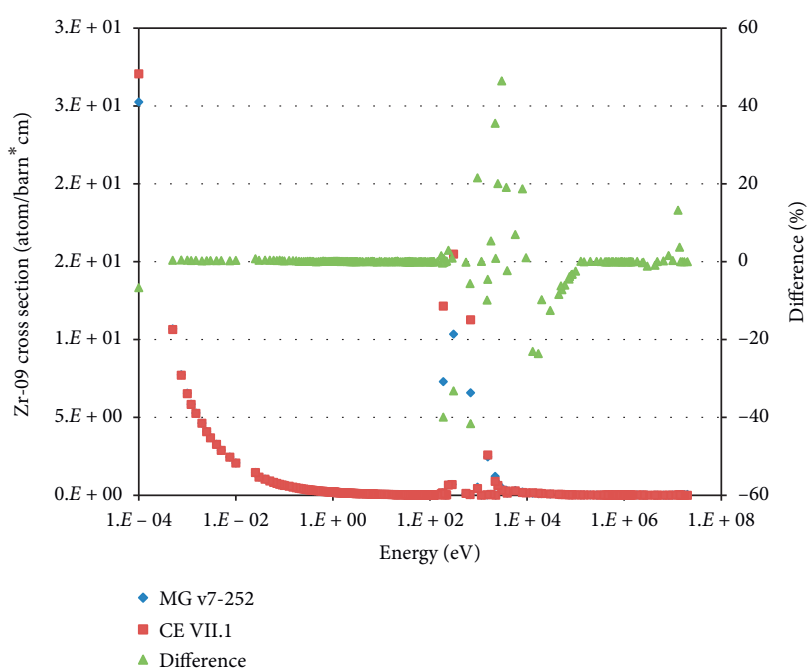

(b)

Figure 10: The postprocessed cross sections of $\mathrm{Hf}^{178}$ (a) and $\mathrm{Zr}^{90}$ (b).

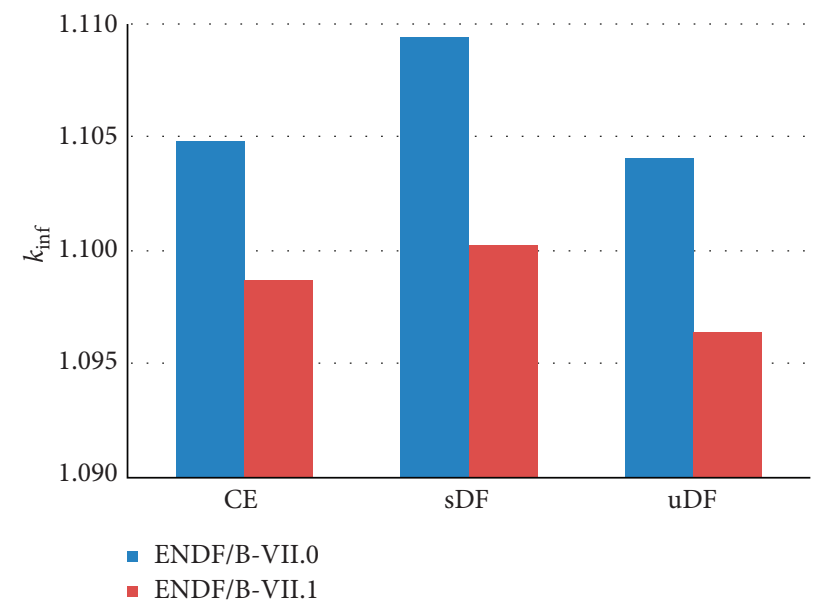

Figure 11: Neutron multiplication factor for CE and MG cases.

values of neutron multiplication factor for cases with libraries compiled of ENDF/B-VII. 1 data (CE and MG) are notably lower (around 0.5-0.9\%) compared to ENDF/B-VII. 0 data.

The decomposition analysis performed allowed us to explain the differences in ENDF/B data libraries versions. It should be noted here that, of course, the differences could only be related to the updated cross-section data, as the only difference found between the simulations is the version of nuclear data. Therefore, only the difference in cases with 252 energy groups (ENDF/B-VII.1 data) is demonstrated and analyzed, focusing on the CE case. Results of the decomposition analysis indicated that the differences in reactivity are mainly influenced by the change of fractional absorption in the graphite region (Figure 12(a)). All other differences are associated with variations of neutron fluxes due to the changed neutron absorption rate in the graphite where, after the update, the data of cross sections were employed.
Although only the nonsignificant part of neutrons is absorbed in graphite, the relative change in fractional absorption value is the most considerable: it increases from $3.24 \%$ for the v7.0 version to $3.67 \%$ for the v7.1 version (see Figure 4 ). The decomposition analysis of $\Delta \rho_{\text {CE7.0-CE7.1 }}$ to separate energy groups revealed the significant increase of neutron absorption in thermal and epithermal energy range $(0.025-0.25 \mathrm{eV})$ for the updated version of the library (Figure 12(b)). Thus, lower values of the multiplication factor for simulations with the v7.1 library are related to higher absorption cross-section data in this energy range in comparison with the v7.0 data.

The graphite neutrons absorptions cross sections used in the simulations and generated from ENDF/B-VII. 0 and ENDF/B-VII.1 CE libraries are presented in Figure 13. The data for temperatures of $700 \mathrm{~K}$ and $800 \mathrm{~K}$ are presented as the temperature of $750 \mathrm{~K}$ for graphite was assumed in simulations. Previous studies $[15,16]$ recorded that some 


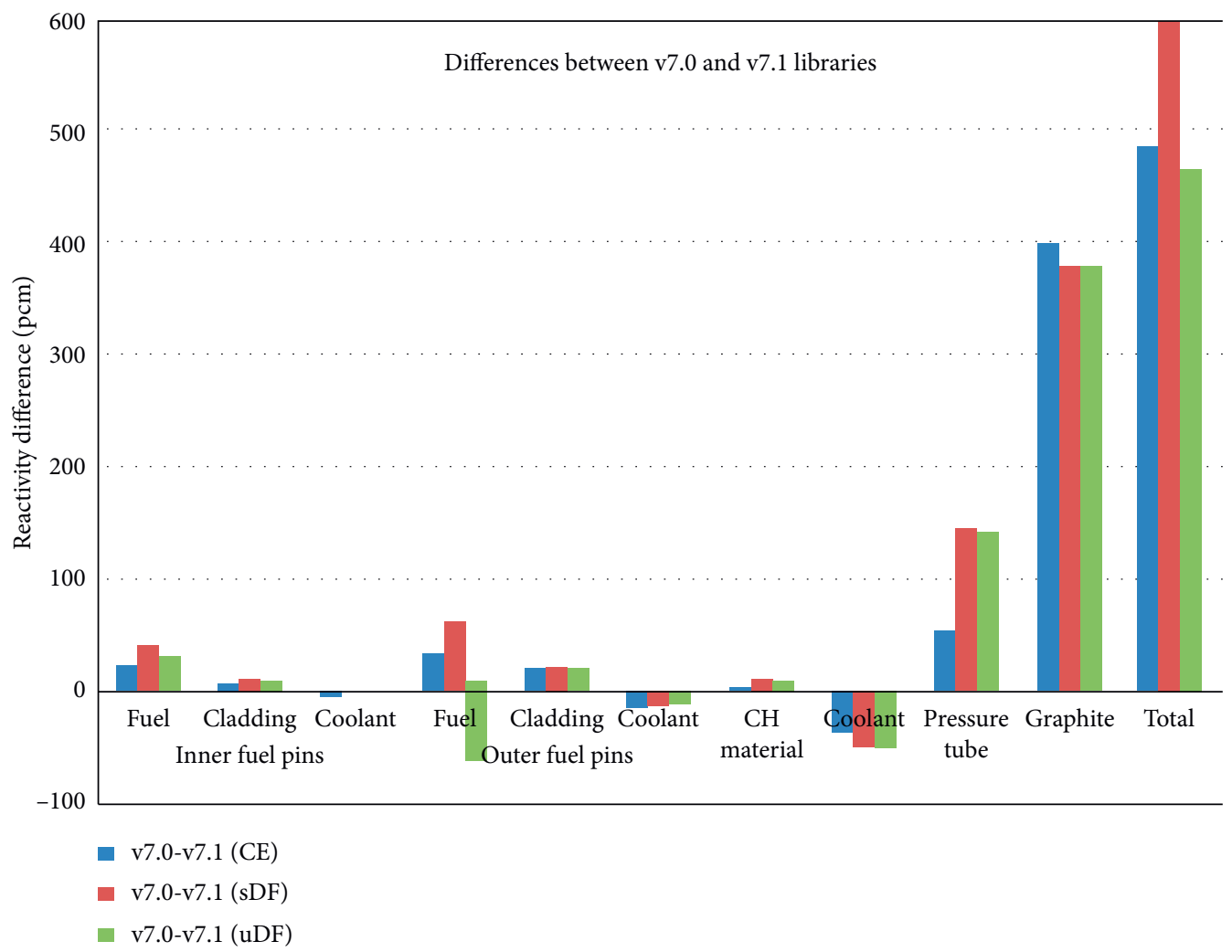

(a)

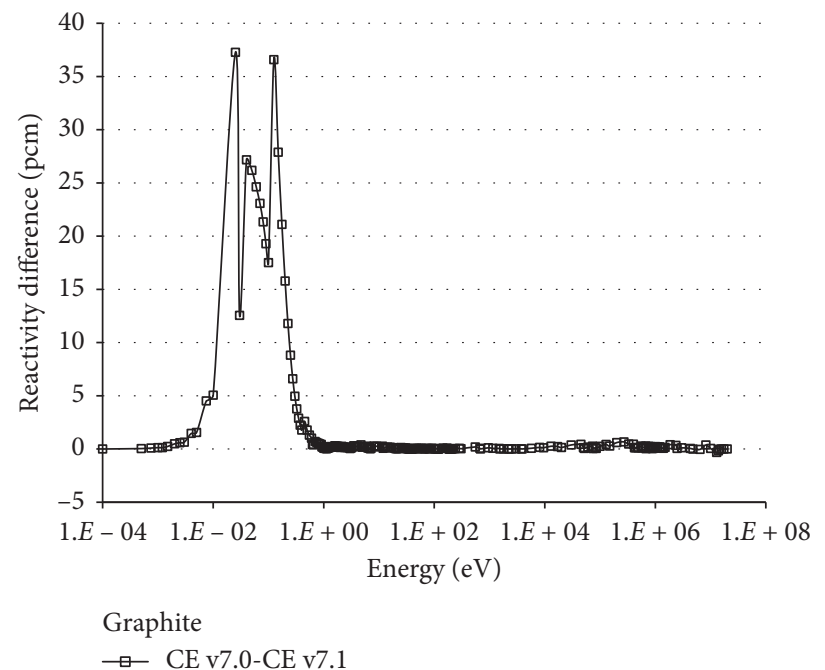

(b)

Figure 12: $\Delta \rho_{\text {CE7.0-CE7.1 }}$ decomposed for separate regions (a) and for separate energy groups in graphite (b).

systematic discrepancies in the reactivity estimations were determined in cases of applying ENDF/B-VII.0 data library to analyze the graphite region considered as a moderator.
Such deflection in the energy range relevant to RBMK, that is, thermal-epithermal range, was determined in this study as well (Figure 13). Improved compliance with the measured 


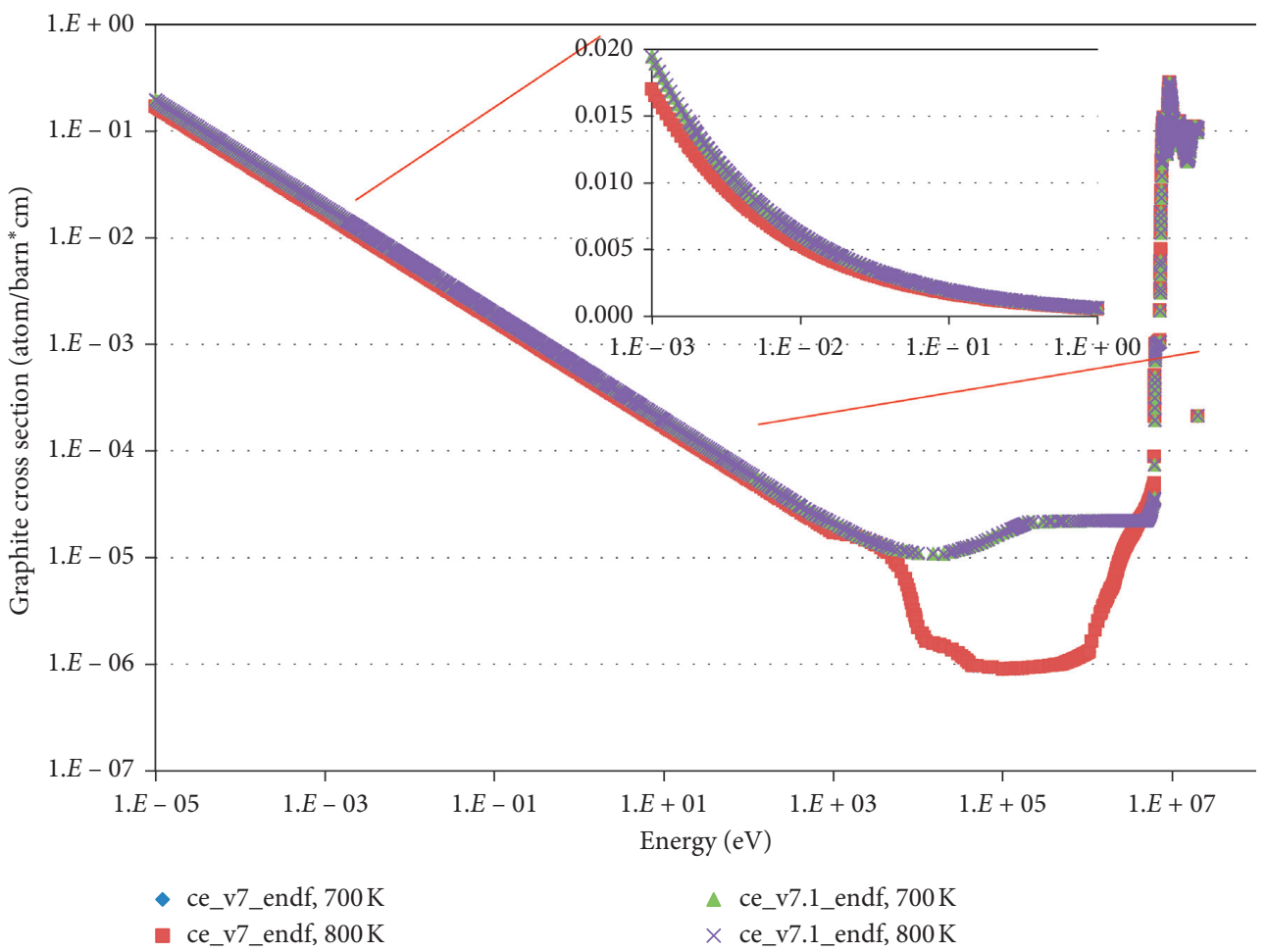

FIGURE 13: Neutron absorption cross sections in the whole energy range.

data when using the latest ENDF cross-section library release, namely, the ENDF/B-VII.1 library, has also been described in [16].

\section{Conclusions}

The neutron transport simulations were performed for the RBMK-1500 fuel assembly in order to investigate available options of resonance processing models and to evaluate the applicability of different cross-section data libraries with the SCALE 6.2.3 code. The neutron transport simulations included the application of both multigroup (MG) and pointwise CE libraries from ENDF/B-VII.0 and the updated ENDF/B-VII.1 evaluated data files.

The results of the investigation on a more detailed resonance processing model showed that the application of user-defined DFs (uDFs) affects the decrease of reactivity in fuel assembly by $455 \mathrm{pcm}$ and $317 \mathrm{pcm}$ for v7-238 and v7252 data libraries, respectively. As expected, the results of the performed decomposition analysis indicated that reactivity changes are influenced mainly by the increase of neutron absorption in the resonance energy range, due to the reduction of DF which directly affects the decrease of resonance escape probability.

The comparison of $\mathrm{uDF}$ results with the reference simulation using CE data libraries showed that the determined reactivity was underpredicted by 60 and $226 \mathrm{pcm}$ for v7-238 and v7-252 data libraries, respectively. In the case of MG data application, the following decomposition analysis disclosed that the discrepancies originated from the overprediction of pressure tube cross sections. Some additional discrepancies of neutron absorption in the fuel region were found for v7-252 data library in the energy range of 20-120 eV. The performed comparison of the results showed that fuel resonance cross sections are not processed correctly enough in this energy range. The resonance processing with the application of the v7-238 data library delivered more accurate results considering the smaller deviation from the reference simulation.

The discrepancies between ENDF/B-VII.0 and ENDF/BVII.1 evaluated data-based libraries were indicated by analyzing the change of the neutron multiplication factor. It was proven that the discrepancies are related to the updated graphite cross sections. The neutron capture rate increases in the graphite block and the neutron multiplication factor decreases with the application of ENDF/B-VII.1 evaluated data-based libraries. Thus, the neutron multiplication factor is overpredicted when applying the v7-238 data library. On the other hand, the use of the v7-252 data library generates a higher deviation from the reference CE simulation due to the resonance processing.

Finally, the selection and usage of MG libraries for the neutron transport simulations of RBMK-1500 fuel assembly must be chosen carefully. The CE library based on ENDF/BVII.1 evaluated data proves to be the best option as it gives the most accurate results on the analyzed system reactivity.

The neutron transport simulations, being an integral part of irradiation calculations, remain an issue to be considered during the estimations of spent fuel characteristics. Further studies are focused on investigating the effect of a more 
detailed resonance processing model on RBMK spent fuel characteristics.

\section{Data Availability}

The data are available upon request to the authors.

\section{Conflicts of Interest}

The authors declare that there are no conflicts of interest regarding the publication of this paper.

\section{References}

[1] Scale: A Comprehensive Modeling and Simulation Suite for Nuclear Safety Analysis and Design, ORNL/TM-2005/39, Version 6.1, Radiation Safety Information Computational Center at Oak Ridge National Laboratory as CCC-785, June 2011.

[2] S. M. Dancoff and M. Ginsburg, Surface Resonance Absorption in a Close-Packed Lattice, CP-2157, United States Atomic Energy Commission, Technical Information Service Extension, Washington, DC, USA, 1944.

[3] B. J. Ade, SCALE/TRITON Primer: A Primer for Light Water Reactor Lattice Physics Calculations, ORNL/TM-2011/21, Oak Ridge National Laboratory, Oak Ridge, TN, USA, 2012.

[4] H. Smith, "Modeling depletion simulations for a high-burnup, highly heterogeneous BWR fuel assembly with SCALE," in PHYSOR-Advances in Reactor PhysicsAmerican Nuclear Society, Chicago, IL, USA, 2012.

[5] C. Mesado, D. Morera, R. Miro et al., "Comparison of depletion results for a boiling water reactor fuel element with CASMO and SCALE 6.1 (TRITON/NEWT)," in Proceedings of the INAC-International Nuclear Atlantic Conference, Recife, Brazil, November 2013.

[6] B. J. Marshall, B. Ade, S. M. Bowman et al., "Technical basis for peak reactivity burnup credit for BWR spent nuclear fuel in storage and transportation systems," Office of Nuclear Regulatory Research, Washington, DC, USA, ORNL/TM-2014/ 240, 2015.

[7] I. C. Gauld and U. Mertyurek, "Validation of BWR spent nuclear fuel isotopic predictions with applications to burnup credit," Nuclear Engineering and Design, vol. 345, pp. 110-124, 2019.

[8] M. I. Radaideh, D. Price, D. O'Grady, and T. Kozlowski, "Advanced BWR criticality safety part I: model development, model benchmarking, and depletion with uncertainty analysis," Progress in Nuclear Energy, vol. 113, pp. 230-246, 2019.

[9] J. Cardoni and Z. Jankovsky: Fukushima Daiichi Radionuclide Inventories, SAND2016-9065R, September 2016.

[10] R. Plukienè, A. Plukis, D. Germanas, and V. Remeikis, "Numerical sensitivity study of irradiated nuclear fuel evolution in the RBMK reactor," Lithuanian Journal of Physics, vol. 49, no. 4, pp. 461-469, 2009.

[11] K. Almenas, A. Kaliatka, and E. Uspuras, Ignalina RBMK1500. A Source Book, Lithuanian Energy Institute, Kaunas, Lithuania, 1998.

[12] B. D. Murphy, ORIGEN-ARP Cross-Section Libraries for the RBMK-1000 System, Oak Ridge National Laboratory, ORNL/ TM-2006/, Nashville, TN, USA, 2006.

[13] G. Ilas, B. D. Murphy, and I. C. Gauld, "Overview of ORIGENARP and its applications to VVER and RBMK," Transactions of American Nuclear Society, vol. 97, pp. 601-603, 2007.
[14] A. Slavickas, R. Pabarčius, A. Tonkūnas, G. Stankūnas, and E. Ušpuras, "Void reactivity coefficient analysis during void fraction changes in innovative BWR assemblies," Science and Technology of Nuclear Installations, vol. 2015, Article ID 757201, 8 pages, 2015.

[15] M. Koštal, V. Rypar, J. Milčák et al., "Study of graphite reactivity worth on well-defined cores assembled on LR-0 reactor," Annals of Nuclear Energy, vol. 87, pp. 601-611, 2016.

[16] F. Bostelmann, H. R. Hammer, J. Ortensi, G. Strydom, K. Velkov, and W. Zwermann, "Criticality calculations of the very high temperature reactor critical assembly benchmark with serpent and SCALE/KENO-VI," Annals of Nuclear Energy, vol. 90, pp. 343-352, 2016. 